\title{
Research on PV Array Voltage Characteristic Test System Design
}

\author{
Feng Fei ${ }^{1, \mathrm{a}}$, Wu Fangsheng ${ }^{1, \mathrm{~b}}$, Chen Jianfu ${ }^{2, \mathrm{c}}$, Wang Weishan ${ }^{2, \mathrm{~d}}$ \\ ${ }^{1}$ Changzhou Institute of Engineering Technology, Jiangsu, Changzhou, 213164 \\ 2 Jiangsu University of Technology, Jiangsu, Changzhou, 213000 \\ ${ }^{a}$ email, ${ }^{b}$ email, ${ }^{c}$ email, ${ }^{d}$ email
}

KEYWORDS: PV Array; Voltage Characteristic; Test System; Design

\begin{abstract}
Solar photovoltaic power generation is an important part of the system, and the work efficiency and system capacity is closely related to improving the energy conversion efficiency of the solar cell can increase the utilization of solar energy. Because of the characteristics of solar cells have a number of factors affecting the practical application, therefore, when configuring the array of photovoltaic cells, the need to test its characteristics, select the cell array to meet the requirements, improve the efficiency of photovoltaic power generation system. By analyzing the volt-ampere characteristics and testing requirements for photovoltaic cell array, is designed to test the system, discusses the overall system architecture, and system testing principle was studied briefly analyzes the main part of the system functions.
\end{abstract}

\section{Introduction}

Solar energy is a clean, renewable energy development and utilization prospects. Use of solar energy includes a light-heat conversion, a light-electricity conversion and light a chemical conversion in three ways. Solar photovoltaic power generation is an important part of the system, has a significant impact on its efficiency and power output. By improving the performance and stability of the solar energy conversion, can greatly increase the use of solar energy. Compared with the conventional thermal power, there is no danger of photovoltaic energy shortages and transportation restrictions, the roof and other buildings can be used in situ generation, energy obtain fast and high-quality, safe, easy exploitation. Power for exploitation in many areas, and promote the photovoltaic related products research and production, to ease the energy shortage and reduce energy consumption to environmental hazards indicates a new direction based on these facilities, photovoltaic. Solar photovoltaic power generation is the primary means of development and utilization of pounds, is an important component of photovoltaic power generation system has a significant impact on its efficiency and power output. Therefore, the installation of solar photovoltaic power generation system suitable for use, and improve its performance, has been a key research photovoltaic technology. Through the relevant characteristics of analysis and testing of solar cells, can further increase its efficiency and improve the working efficiency of the photovoltaic power generation systems, while reducing the cost of photovoltaic power generation.

\section{The Test System Design}

Solar cell characteristics of conventional testing methods typically can vary the resistance of the resistance as the load and into the output circuit of the solar cell. When the solar cell to power the load, before the circuit is opened, the circuit voltage and current values are constantly changing, you 
can use a voltmeter and ammeter respectively, and other equipment to test samples of these data, and the calculation and analysis, thereby obtaining the solar battery output voltage characteristics curve. However, due to changes in load resistance is not continuous, and the accuracy of the sampling device is relatively low, these factors will affect the test results of the solar cell characteristics.

Characteristics of the test array of photovoltaic cells are a change in the voltage, the current value of the sampling process. As a capacitive load on the battery voltage and current signals output from the array is sampled, the sampling time can reduce test. During array of photovoltaic cells to charge the capacitor, since capacitor discharge effect, the circuit voltage and current magnitude changing until the end of the charging process, and does not change in one part of the load resistance value is not continuous, so that sampled data is abnormal. Further, the capacitive load as a photovoltaic cell array, in theory, the equivalent load resistance can be increased from zero to infinity, and the traditional use of variable resistance as the battery load, but cannot increase its resistance to infinity, the exact impact of the test sample, the use of capacitive loads as a photovoltaic cell array, can be relatively accurate testing sample data.

\section{The Main Part Features of the System}

DSP system control unit via the system function requirements analysis and system design, as used herein TMS320F2806DSP chip control system to achieve the overall control of the testing system. Structural Systems DSP control unit mainly includes partial control chip, sampling control circuit, driving circuits. DSP peripheral circuit components of the control unit by the minimum system power supply, ADC unit and the connection of the JTAG interface and the like.

Capacitor charge and discharge circuit can be determined by the magnitude of the capacitance of the capacitor array of photovoltaic cells for charging time will also affect the rate and volume of the sampling system. When the capacitance value is larger when the capacitance between the charge-inch long, beneficial system for data sampling test, however, the volume of the system becomes large; when the capacitance value is small time, although you can reduce the volume of the test system, but it would also reduce the capacitor charging time, the number of influence of sampling data, so need to choose the appropriate capacitance values.

Voltage and current sampling system were designed voltage and current sampling circuit, the capacitor charging voltage and current signals are sampled. Through the analysis, the system selects the resistance greater than $1 \mathrm{M}$ precision high value resistor dividing realize the voltage signal sampling and dividing resistor flowing current is very small, and will ignore the latter will not affect its current signal system sampling. Voltage divider resistor voltage signal sampling time, the voltage $0-600 \mathrm{~V}$ can be converted into the sampling signal $0-5 \mathrm{~V}$. According to the system requirements, preclude the kind of current range 0-100A, the system uses current sensor array of photovoltaic cells on the current signal output is sampled, which preclude the kind of time is short, fast and wide. So choose 100A / 4V of HDC100LB Hall sensor, the error is small enough to ensure that the data is accurate, that the sampling system requirements.

As used herein, DS18B20 temperature sensor test system like when complete the system requirements for temperature data sampling, and sampling data obtained by the A / D conversion process, the temperature can be programmed to signal into digital bits 9-12 signals, the communication interface chip converts the temperature sample data to the control unit DSP system.

System selection HG230240 touch screen as a display unit, HG230240 dot number of 320X240, blue / gray display mode, the supply voltage is $5 \mathrm{~V} / 3.3 \mathrm{~V}$, and selected with temperature compensation, it is possible to achieve independence and combination of graphics or text display, 
and use the control unit to control it. Since the DSP chip used herein contain only serial communication interface (SCI), so the test system by using RS-485 interface on the sampling data transmission system requirements.

\section{The Selection of Array Photovoltaic Cells Characteristic Test Method}

Solar photovoltaic systems generally work under the sun light intensity and temperature constantly changing conditions, and characteristics of the photovoltaic cell array power generation efficiency and performance of the entire system has a relatively large impact. In order to get accurate output voltage characteristics of the photovoltaic cell array, according to the International Electro-technical Commission requirements for test conditions, the standard test conditions herein set the system are: solar radio flux blessing $1000 \mathrm{~W} / \mathrm{m} 2$, ambient temperature of $25^{\circ} \mathrm{C}$, the atmosphere quality AMI.5 (AM to air quality).

The method is typically used to connect a variable resistor for voltage characteristic of the test array of photovoltaic cells, which is to select a variable resistor as the load of the array of photovoltaic cells, and the voltage meter and test access circuit, FIG. 3-5 is schematic variable resistance test method. By controlling the resistance is changing, the process of change in resistance, respectively voltage and current values in the circuit, and the test data obtained through an array of photovoltaic cells to calculate the output voltage characteristic curve.

The use of electronic load is applied to the array of photovoltaic cells connected MOS transistor and the compensation circuit composed of power as part of its load resistance, by controlling the size of the electronic load equivalent resistance from zero to infinity, this method is the electronic load test method, resistance to changing the size of the sample during the test array of photovoltaic cells connected voltage and current values in Road, according to the volt-ampere characteristics of these data calculated PV array output curve.

By analyzing the effect of capacitor charging and discharging, a certain capacity capacitor connected to the output circuit array of photovoltaic cells as part of their load. Array of photovoltaic cells in the process of charging the capacitor, the capacitance changing voltage and current, by sampling voltage and current values can be obtained an output voltage characteristic curve of the photovoltaic cell array, this method is dynamic charging capacitor test method, whereby voltage characteristics of PV array output for testing.

\section{The Hardware and Software Design of the Test System}

Therefore, the need for effective protection method, when the system is abnormal or faulty, the circumstances in which the alarm and signal is processed and appears to ensure safe operation. In this paper, there are several ways to protect the system. Composed of hardware system is mainly composed of an array of photovoltaic cells for charging and discharging the capacitor circuit, the data processing unit, a control unit, IGBT drive circuit, power supply system, the system displays and other components. Dynamic test system based on capacitive charging test method, the test system using IGBT to achieve capacitance charging and discharging switch control circuit; power supply system includes a power supply requirements of multi-channel power output, to meet the various hardware parts of the circuit, effectively ensure the security of the system and handling; sampling circuit is the main circuit test system hardware, to achieve the output voltage and current of photovoltaic cell array, solar light intensity and temperature data test sample, and calculating the data analysis by the data processing unit, while its conversion processing; vehicle control element 
using the DSP chip for overall system control; display unit complete system test data output display, using the RS-485 data communication transmission.

System software is part of the main process control data sampling, and sampling data points Watchman and processing, display output unit sampling conditions and results through the system, to the time when the system is abnormal situation, to take measures to protect the system.

\section{Conclusion}

By studying the relevant characteristics of the solar cell, the mathematical model of the battery, and refer to the actual application, the model was simplified, results calculated battery voltage characteristics and select the arithmetic mean of the digital filter sampling method for data processing. Analysis and comparison, to determine the dynamic charging the capacitor array of photovoltaic cells as a test method Test methods for characterizing, and testing methods are analyzed in detail. According to system design and dynamic capacitance charging test method, design the overall structure of the system hardware, the main part of the circuit and functional overview. Select DSP chip system as the system control unit via parameter calculation and device selection, design capacitive charge-discharge circuit hardware of the system, the data sampling circuit, IGBT drive circuit and display circuit and method for analysis of protection systems. The system features a hardware circuit and software requirements, analysis and design of the main test, data sampling program, digital filtering routines, display the software part of the program, the system abnormality protection, etc., and the basic function of each part and the specific processes discussed.

\section{Acknowledgements}

Project: Changzhou Technology Support Program Fund, Project NO. CE20150063

\section{REFERENCE:}

[1] John SteVens, Russel Bonn, Jerry Giiin, Sigifredo Gonzalez. Development and Testing of an Approach to Anti-Islanding in Utility-Interconnected Photovoltaic Systems, 2000.

[2] Green MA,Emery K,King DL, Igari S,Warta W. Solar cell efficiency tables (version 29) [J]. Progress in Photovoltaics: Research and Applications, 2006, 12: 736-741.

[3] Bolko von Roedern. Status of amorphous and crystalline thin film silicon solar cell activities. NCPVAND Solar Program Review Meeting, 2003.

[4] Guha S,Yang J and Banejee. A Amorphous silicon alloy photovoltaic research -present and future. Prod. Photo voltaics, 2000, 8(141).

[5] Altas. A Photovo Raic. Array Simulation Model for Matlab-Simulink GUI Environment [J]. Clean Electrical Power, 2007: 341-345. 